\title{
Results of the fifth international spectroradiometer comparison for improved solar spectral irradiance measurements and related impact on reference solar cell calibration.
}

\author{
R. Galleano ${ }^{1}$, W. Zaaiman ${ }^{1}$, D. Alonso-Álvarez ${ }^{2}$, A. Minuto ${ }^{3}$, N. Ferretti ${ }^{4}$, R. Fucci ${ }^{5}$, M. \\ Pravettoni $^{6}$, M. Halwachs ${ }^{7}$, M. Friederichs ${ }^{8}$, F. Plag ${ }^{9}$, D. Friedrich ${ }^{9}$, E. Haverkamp ${ }^{10}$ \\ ${ }^{1}$ EC, DG JRC, IET, Renewables and energy efficiency Unit, ESTI, roberto.galleano@ec.europa.eu, Ispra, (I), ${ }^{2}$ EXSS group, Department \\ of Physics, Imperial College, London, (UK), ${ }^{3}$ RSE spa, Milan, (I), ${ }^{4}$ Photovoltaik-Institut Berlin, (D), ${ }^{5}$ ENEA Research Center of Portici, \\ (I), ${ }^{6}$ SUPSI, Campus Trevano, CH-6952, Canobbio, (CH), ${ }^{7}$ AIT, Austrian Institute of Technology, 1210 Vienna, (AT), ${ }^{8}$ PV Lab \\ Germany, 14482 Potsdam, (D), ${ }^{9}$ Physikalisch-Technische Bundesanstalt, Arbeitsgruppe "Solarzellen", 38116 Braunschweig, (D), \\ ${ }^{10}$ Radboud University Nijmegen, AMS, IMM, Heyendaalseweg 135, 6525 AJ Nijmegen, (NL)
}

\section{Introduction}

The wider portfolio of today's available photovoltaic (PV) technologies on the market makes the measurement of the spectral content of the natural or simulated sunlight a key parameter for the characterization, calibration, and energy yield estimation of these devices. Nowadays, spectroradiometers with different principles of operation (e.g. single-, doublestage rotating grating monochromator or fixed single grating polychromator with photodiode (PD) array or CCD detectors) are routinely used for solar spectral irradiance measurements. At present there is a growing request for harmonization of good measurement practices and for knowledge transfer in the field of spectrally resolved solar radiation for solar energy applications (e.g. photovoltaics) in order to make these measurements comparable and directly traceable to SI units. Moreover, periodical round robin or intercomparisons are part of performance-based quality-control checks for laboratories working according to ISO-IEC 17025 [1] standard.

Within this context, a group of European research institutes active in the PV field for research, characterization and engineering, set up the fifth comparison of spectroradiometers for solar spectral irradiance measurements. Aims of the intercomparison were: to assess laboratory measurement capabilities; to exchange and compare instrument calibration procedures; to establish equivalence figures for the measurement of solar spectra; to put in practice lessons learnt from previous editions; and to evaluate the impact the comparison results may have on a practical case. This paper describes the intercomparison campaign, reports on the results and analyses the impact these results would have on the primary calibration of a c-Si PV reference cell under natural sun light.

\section{Purpose of the work, experimental approach}

There is a growing request for harmonized, traceable and low uncertainty solar spectrum measurements for calibration and energy yield estimation in PV. This intercomparison was designed to raise the awareness and exchange good practices on reliable, traceable measurements of the solar spectral irradiance with low uncertainty. Moreover, for the participating institutes applying a quality system or having an accreditation according to the ISO/IEC17025 standard [1], the comparison is an implementation, together with round robin, of the required checks for establishing a performance-based quality-control system. The intercomparison took place for the second time at the 'Instituto Nacional de Técnica Aeroespacial' laboratory, near Madrid (Spain) from $18^{\text {th }}$ to $22^{\text {nd }}$ of May 2015. Twelve spectroradiometers systems from six different manufacturers and covering two different technologies (single-stage rotating-grating and fast fixed-grating polychromator with single 
PD or CCD array detectors) were set to simultaneously measure the global normal incidence (GNI) spectral irradiance of natural sun light from 300 to $1700 \mathrm{~nm}$ or 300 to $1100 \mathrm{~nm}$, depending on the available instruments.

The large variety of manufacturers and principles of operation represent a good cross-section of today's most used spectroradiometers in the PV community. A selected number of instruments, equipped with suitable collimation tubes to reduce their angle of view [2], were set to measure also the direct normal incidence (DNI) spectral irradiance in the range from 300 to $1700 \mathrm{~nm}$. In this paper only the GNI measurements results from the ten laboratories successfully measuring will be reported.

Due to the technical differences among various instruments in the measurement timing, bandwidth and spectral resolution, specific procedures for data acquisition, synchronization and analysis were developed in order to make the spectroradiometers' output data comparable to each other. Data processing procedures are summarized below and described in more detail elsewhere [3-4].

Prior to the intercomparison, each participating laboratory calibrated their own spectroradiometer(s) following their usual procedures, thus allowing evaluating the instrument performance together with its traceability chain and calibration procedure. Some spectroradiometers were calibrated by an external accredited calibration laboratory, while others were calibrated in-house using a calibrated radiometric standard lamp, or at the manufacturer.

Table 1 summarizes the main characteristics of the instrument involved: commercial name, instrument type (poly- or mono- chromator and detector configuration), the wavelength range covered, and the calibration source. All participating instruments were mounted on high-accuracy $\left(0.5^{\circ}\right.$ pk-to-pk) solar trackers in order to reduce errors due to instruments pointing (e.g. different cosine response of the instruments' entrance optic). In parallel to the intercomparison a set of cavity radiometers were used as reference detectors for total irradiance data. These last ensure the direct link of solar irradiance measures to the SI units as these cavity radiometers take part to the international pyrheliometer comparison under the aegis of the World Radiometric Reference (IPC - WRR), held every 5 years at PMODDavos $(\mathrm{CH})[5]$.

\begin{tabular}{|c|c|c|c|c|}
\hline Laboratory & Instrument name & Instrument type & $\begin{array}{l}\text { Wavelength band } \\
\mathrm{nm}\end{array}$ & $\begin{array}{l}\text { Calibration } \& \\
\text { traceability path }\end{array}$ \\
\hline AIT & $\begin{array}{l}\text { Ocean Optics } \\
\text { USB2000, NIR Quest }\end{array}$ & $\begin{array}{l}\text { Polychromator, two } \\
\text { CCD array detector }\end{array}$ & $300-1600$ & $\begin{array}{l}\text { In house, standard } \\
\text { lamp }\end{array}$ \\
\hline ENEA & $\begin{array}{l}\text { Stellarnet, EPP2000 } \\
\text { UVN-NIR }\end{array}$ & $\begin{array}{l}\text { Polychromator, two } \\
\text { CCD array detectors }\end{array}$ & $300-1700$ & $\begin{array}{l}\text { External accredited } \\
\text { cal. lab. }\end{array}$ \\
\hline $\begin{array}{l}\text { Imperial } \\
\text { College }\end{array}$ & $\begin{array}{l}\text { Ocean Optics } \\
\text { HR } 4000\end{array}$ & $\begin{array}{l}\text { Polychromator, CCD } \\
\text { array detector }\end{array}$ & $250-1100$ & In house calibration \\
\hline $\mathrm{JRC}$ & OL750 & $\begin{array}{l}\text { monochromator } \\
\text { double PD detector }\end{array}$ & $250-2500$ & $\begin{array}{l}\text { In house, standard } \\
\text { lamp }\end{array}$ \\
\hline PI Berlin & Tec5, tecSpec & $\begin{array}{l}\text { Polychromator, CCD } \\
\text { array detector }\end{array}$ & $300-1700$ & $\begin{array}{l}\text { In house, standard } \\
\text { lamp }\end{array}$ \\
\hline РTB & $\begin{array}{l}\text { Instrument } \quad \text { System } \\
\text { CAS 140CT 156-171 }\end{array}$ & $\begin{array}{l}\text { Polychromator, three } \\
\text { CCD array detectors }\end{array}$ & $250-2150$ & $\begin{array}{l}\text { In house, standard } \\
\text { lamp }\end{array}$ \\
\hline
\end{tabular}




\begin{tabular}{|c|c|c|c|c|}
\hline RSE & Stellarnet EPP2000 & $\begin{array}{l}\text { Polychromator, two } \\
\text { CCD array detectors }\end{array}$ & $300-1700$ & Outdoor, AM1.5 \\
\hline $\begin{array}{l}\text { Radboud } \\
\text { University }\end{array}$ & EKO MS711 & $\begin{array}{l}\text { Polychromator, CCD } \\
\text { array detector }\end{array}$ & $300-1100$ & Manufacturer \\
\hline PV Lab & $\begin{array}{l}\text { Instrument System } \\
\text { CAS 140CT } 156\end{array}$ & $\begin{array}{l}\text { Polychromator, CCD } \\
\text { array detector }\end{array}$ & $300-1100$ & Manufacturer \\
\hline SUPSI & EKO wiser system & $\begin{array}{l}\text { Polychromator, two } \\
\text { CCD array detectors }\end{array}$ & $300-1700$ & $\begin{array}{l}\text { In house, standard } \\
\text { lamp }\end{array}$ \\
\hline
\end{tabular}

Table 1: Summary of the characteristics of the spectroradiometers involved in the intercomparison. Commercial name, principle of operation, measuring wavelength band and calibration methods are reported.

In order to compare solar spectra acquired by 'fast' and 'slow' measuring instruments, several sets of average spectra, measured during 7-minute acquisition time series, were analysed. The duration of the time series was determined by the acquisition time of the slowest spectroradiometer. During each time series, the irradiance must remain stable to $1 \%$, or better, to consider the series 'stable' and flagged for analysis. The stability constraint avoids adding errors arising from fast changing weather or air mass conditions affecting the output of spectroradiometers in different ways. For instance, a cloud rapidly passing close to the sun disk may affect and invalidate only few spectra in the 7-minute series acquired by a fast spectroradiometer while, for slow spectroradiometers, it may results in a spectrum shape distortion and invalidate the whole 7-minute measurement series. This constraint limited the useful sky conditions to clear or almost clear and discarded acquisitions at early morning and late afternoon. Moreover, the acquired spectra were also convoluted using a Gaussian function in order to increase and harmonize the spectral bandwidth to $4 \mathrm{~nm}$ full width half maximum (FWHM); this is done to reduce artefacts when comparing spectra in the atmospheric absorption bands [3-4].

Several analyses were performed on acquired data, both in terms of absolute spectral irradiance and of spectral shape deviations. As described later, diversified data analysis approaches have higher chances of detecting and discriminating errors or uncertainty components due to systematic effects (e.g. instrument calibration) from those arising from instrument non linearity, internal stray light or drifts, as some of these errors or uncertainties might not be evident in all analyses.

\section{Intercomparison results and discussions}

\subsection{Absolute spectral irradiance analysis}

Due to a confidentiality non-disclosure agreement among the participants and to avoid commercialism, results will be presented in an anonymous way. Figures 1 to 3 show a wavelength-by-wavelength (W-by-W) spectral irradiance deviation analysis performed on a set of typical GNI spectra measured by the partners' instruments over two days of intercomparison. Spectra calculated by SMARTS [16] are also included for redundancy and independent check purposes (see Appendix A for details on its use and required inputs).

The graphs in the three figures denoted by letter (a) show a group of stable simultaneously acquired spectra during a 7-minute acquisition series. Graphs (b) in the same figures show the W-by-W percent deviation of each spectrum with respect to Lab A spectrum and normalized to its peak irradiance. The Lab A instrument was chosen as reference because it was one of the instruments calibrated via a metrological unbroken chain to the SI units with 
low uncertainty and a full evaluation of its calibration uncertainty was provided. W-by-W percent deviation data allow inferring some preliminary information about instruments stability during some hours of continuous outdoor measurements and about instruments reproducibility when considering different days of measurement. All but one instruments were dismounted from trackers at the end of each day and sheltered in a nearby laboratory during the night because not specifically designed for permanent outdoor operation.

When considering the entire ensemble of the spectroradiometers, the average W-by-W deviation values for the reported spectra lie in a band of $\pm 2.4 \%$ with associated standard deviations up to $4 \%$ for Figure 1, in a band of $\pm 2.6 \%$ with associated standard deviations up to $5.1 \%$ for Figure 2, and in a band of $\pm 3.4 \%$ with associated standard deviations up to $4.9 \%$ for Figure 3. When considering the behaviour of each single instrument shown in Figure 1 to 3, most of the instruments achieved repeatable (i.e. during the same day), and reproducible (i.e. during different days) deviations to within $0.6 \%$ pk-to-pk (Labs B, D, E, F, G, I), two instruments (Labs $\mathrm{C}$ and $\mathrm{H}$ ) showed deviations to within $2.5 \%$ pk-to-pk, and one instrument ( $\mathrm{Lab} \mathrm{L}$ ) showed a repeatability to within $0.6 \%$ pk-to-pk during one day of measurement. While data on Figures 1 to 3 show W-by-W mean values calculated on three single acquisitions, a further analysis can be made on the daily average of the W-by-W deviations calculated using all the stable spectra acquired during two days of the comparison.
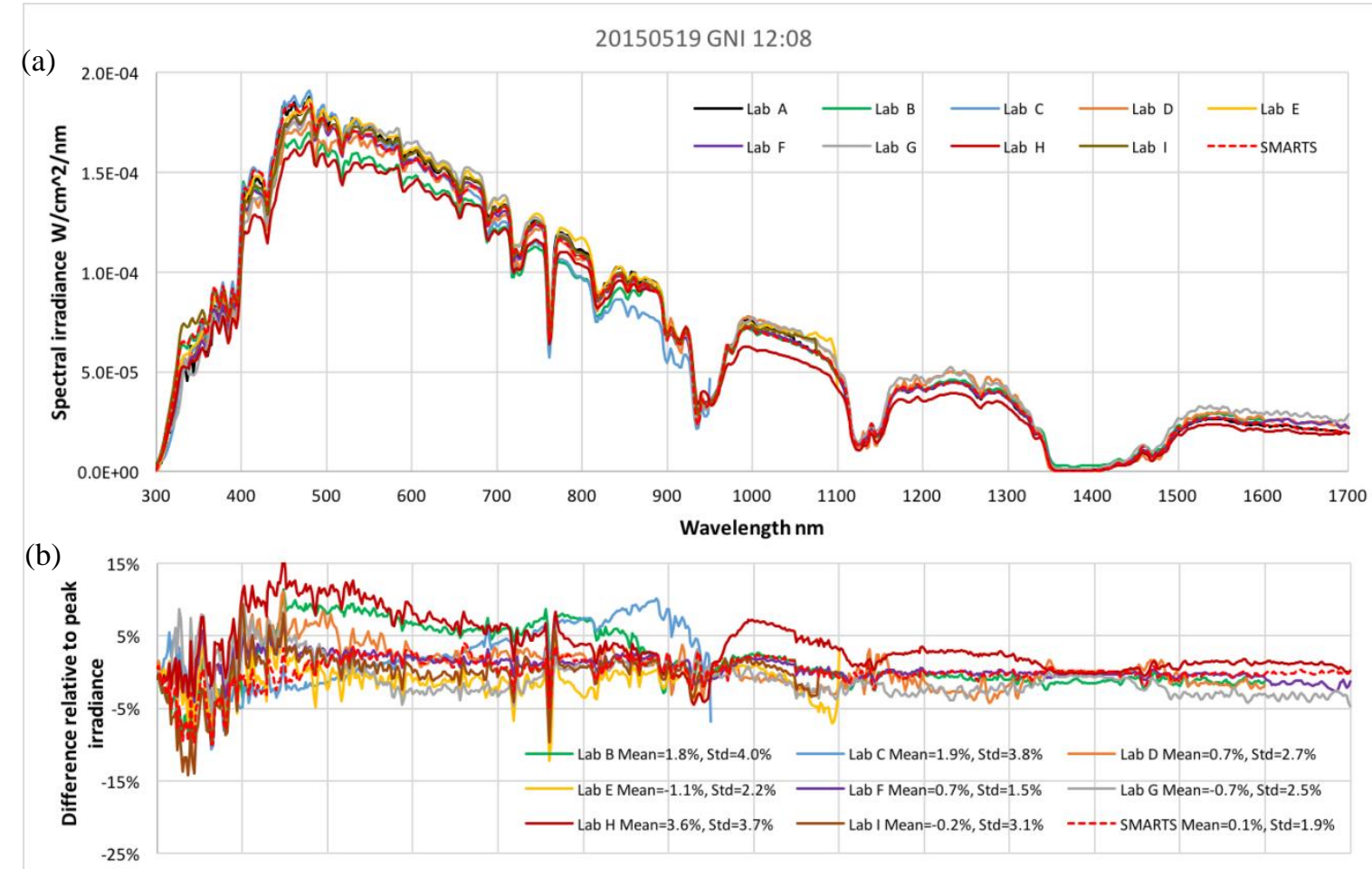

Figure 1: (a) Nine GNI solar spectra simultaneously measured by participating spectroradiometer systems. (b) wavelength-by-wavelength difference of the Lab A spectrum with respect to spectra measured by other laboratories and normalized to Lab A peak irradiance; calculated mean differences and standard deviations are also reported. For comparison purposes calculated SMARTS spectrum is also added. 


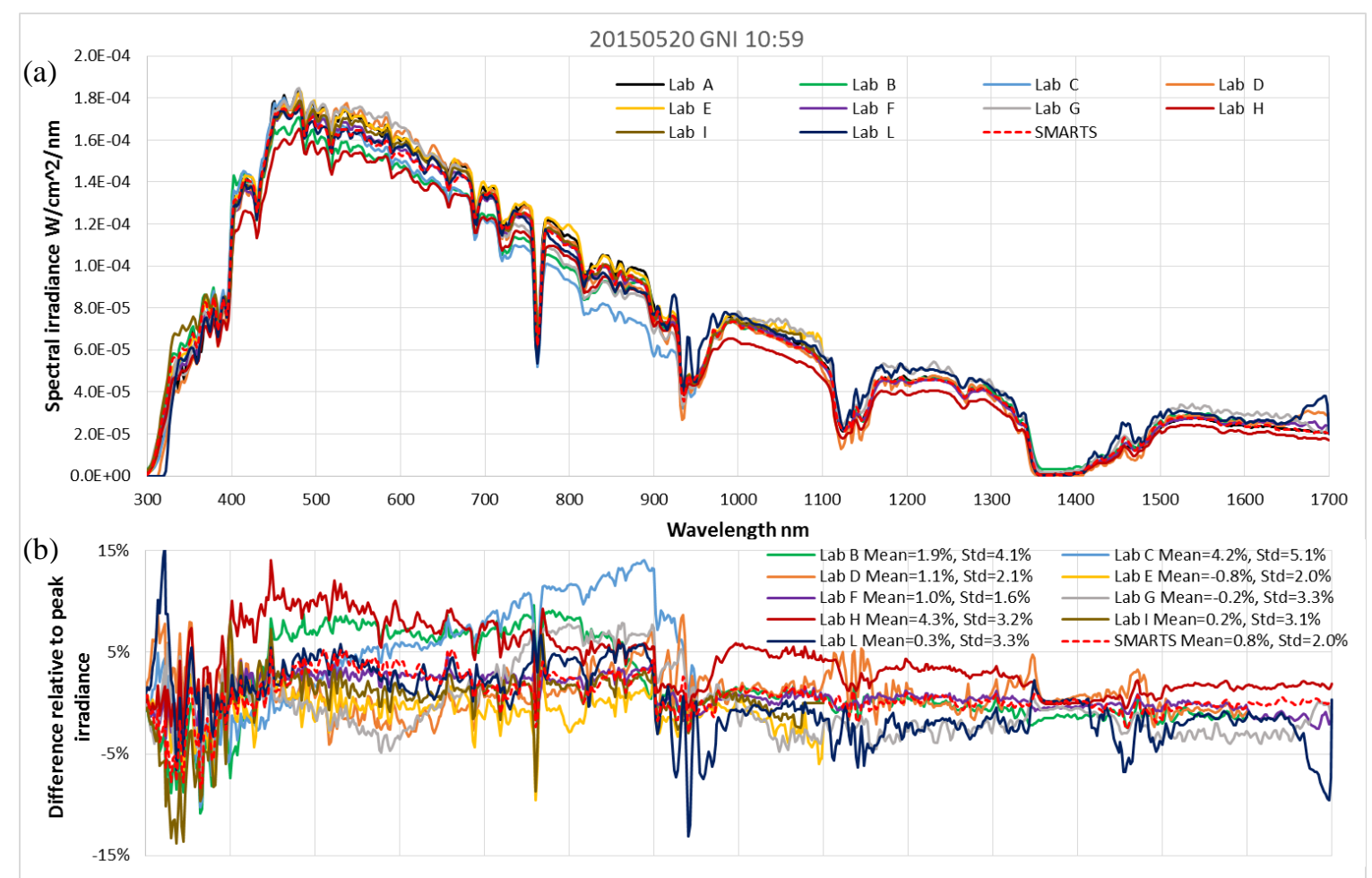

Figure 2: Ten GNI solar spectra simultaneously measured by participating spectroradiometer systems. (b) wavelength-by-wavelength difference of the Lab A spectrum with respect to spectra measured by other laboratories and normalized to Lab A peak irradiance; calculated mean differences and standard deviations are also reported. For comparison purposes calculated SMARTS spectrum is also added.

20150520 GNI 13:17
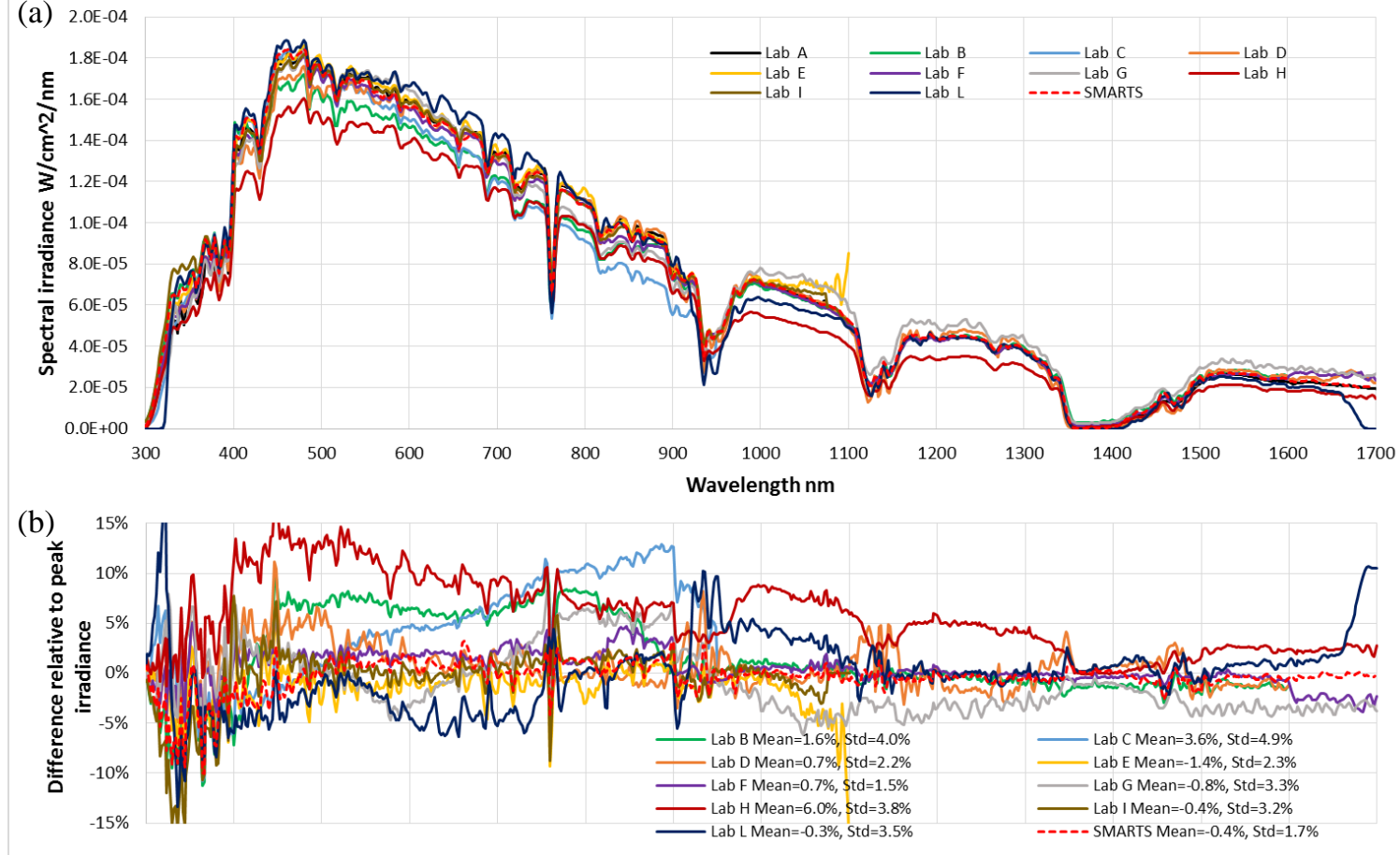

Figure 3: (a) Ten GNI solar spectra simultaneously measured by participating spectroradiometer systems. (b) wavelength-by-wavelength difference of the Lab A spectrum with respect to spectra measured by other laboratories and normalized to Lab A peak irradiance; calculated mean differences and standard deviations are also reported. For comparison purposes calculated SMARTS spectrum is also added. 
Table 2 reports the average $\mathrm{W}$-by-W difference values and their standard deviations for the eight stable GNI spectra measured on May the $19^{\text {th }}$ and the twenty-one stable GNI spectra measured on May the $20^{\text {th }}$. As to May the $19^{\text {th }}$ the average difference values lie within an interval of $\pm 2.7 \%$; similar values were found on May the $20^{\text {th }}$ where the average difference values lie within an interval of $\pm 3.2 \%$. The data labelled as "Lab $\mathrm{C}$ recal" are relative to the recalibration exercise described in chapter 4. A 1-day average standard deviation value much larger than the corresponding 1-day average difference denotes a possible instrument intraday drift and/or instability. From an accurate analysis of this information collected all along the comparison campaign, participants can gain knowledge about their instruments' behaviour such as temperature stability, repeatability and reproducibility.

\begin{tabular}{lrrrr} 
& \multicolumn{2}{c}{$19 / 05 / 2015$} & \multicolumn{2}{c}{$20 / 05 / 2015$} \\
\hline Laboratory & $\begin{array}{r}\text { 1-day Average } \\
\text { difference }\end{array}$ & $\begin{array}{r}\text { 1-day average } \\
\text { std. dev }\end{array}$ & $\begin{array}{r}\text { 1-day Average } \\
\text { difference }\end{array}$ & $\begin{array}{r}\text { 1-day average } \\
\text { std. dev. }\end{array}$ \\
\hline Lab B & $1.9 \%$ & $4.1 \%$ & $1.8 \%$ & $4.0 \%$ \\
Lab C & $3.4 \%$ & $4.2 \%$ & $3.9 \%$ & $5.0 \%$ \\
Lab D & $1.8 \%$ & $2.6 \%$ & $0.8 \%$ & $2.1 \%$ \\
Lab E & $-0.6 \%$ & $2.2 \%$ & $-1.2 \%$ & $2.0 \%$ \\
Lab F & $0.9 \%$ & $1.5 \%$ & $0.8 \%$ & $1.4 \%$ \\
Lab G & $-0.7 \%$ & $2.4 \%$ & $-0.4 \%$ & $3.4 \%$ \\
Lab H & $4.7 \%$ & $4.1 \%$ & $5.1 \%$ & $3.7 \%$ \\
Lab I & $1.1 \%$ & $3.3 \%$ & $-0.3 \%$ & $3.1 \%$ \\
Lab L & & & $-0.2 \%$ & $3.3 \%$ \\
Lab C recal. & $0.1 \%$ & $1.1 \%$ & $0.5 \%$ & $0.6 \%$
\end{tabular}

Table 2: 1-Day average wavelength-by-wavelength differences and standard deviation values calculated with respect to Lab A spectral irradiance and expressed in percentage of its peak irradiance. Reported data refer to two groups of eight and twenty one stable spectra acquired during 7-minute time series on the $19^{\text {th }}$ and $20^{\text {th }}$ of May, respectively. Results from the recalibration of the Lab C instruments are also reported (Cfr. Chapter 4).

\subsection{Relative spectral irradiance analysis}

The previous section focussed on the absolute spectral irradiance differences among participating instruments; a different approach can be used to separate systematic effects (e.g. arising from instrument calibration or from instrument time-drift), from non-linearity or spectra distortion due to intrinsic instrument limitation. This approach is important in solar spectrum measurement applied to PV field, where a correct measurement of the spectral distribution of incoming natural sunlight is fundamental, whereas the absolute irradiance value is usually measured by other means, often with lower uncertainty (e.g. cavity radiometers, reference solar cells, pyrheliometers or pyranometer).

A straightforward comparison of the relative spectral differences among acquired spectra can be done slightly modifying the performance requirements guideline described in [8]. In fact, the aforementioned standard prescribes dividing and integrating the spectral irradiance data of a generic solar simulator into five 100-nm bands from 400 to $900 \mathrm{~nm}$, with an additional 200-nm band from 900 to $1100 \mathrm{~nm}$ and expresses it as percent ratio to its total irradiance as integrated in the 400 to $1100 \mathrm{~nm}$ band. The percentage distribution of irradiance in each band is, then, compared to the same distribution of the AM1.5G standard spectrum to assess its spectral quality class [7], [8].

In this paper the spectral irradiance data of a specific measurement was integrated as 
described before and compared to the same distribution of the Lab A spectrum assumed as reference. As a final step, the $E_{n}$ performance statistics analysis method (for details see Ref. [9]) was applied to the comparison results in each band. The $E_{n}$ number is a performance statistics tool and is defined as:

$$
E_{n}=\frac{M L a b_{i}-M r e f}{\sqrt{\left(U L a b_{i} * M L a b_{i}\right)^{2}+(\text { Uref } * \text { Mref })^{2}}}
$$

Where: $E_{n}$ is the normalized error for the $M_{t h}$ band (dimensionless), $U L a b_{i}$ and Uref are the reported expanded $(\mathrm{k}=2)$ relative measurement uncertainty for the $i_{\text {th }}$ spectroradiometer and the reference, respectively. $M L a b_{i}$ and $M r e f$ represent the ratio between the irradiance of the $M_{t h}$ band to the total irradiance for the $i_{t h}$ spectroradiometer and the reference, respectively.

The calculated $E_{n}$ number in eq. 1 involves the establishment of acceptance limits of \pm 1 ; $E_{n}$ values within acceptance limits are considered satisfactory because consistent with estimated uncertainty. $E_{n}$ values outside acceptance limits highlight inconsistencies with estimated measurement uncertainty and/or severe instrument drift from expected performance. In order to ease the comparison, in this exercise the relative measurements expanded uncertainty $(\mathrm{k}=2)$ were set to a single average value of $5 \%$ for $U L a b_{i}$ and to $3 \%$, resulting from instrument calibration uncertainty, for Uref.

Figure 4 shows, as an example, the performance statistics results as applied to the spectra reported in Figure 2. Six out of nine of the compared instruments show all $E_{n}$ values within the \pm 1 acceptance limits (Labs B, D, E, F, H and I), two instruments (Labs G and L) show one $E_{n}$ value outside acceptance limits, and one instrument (Lab C) has severe deviations from the acceptance limits.

It is worth noticing that the proposed combined data analysis allowed highlighting that Lab $\mathrm{H}$, despite exhibiting the worst W-by-W average difference in Figure 2(b), showed $E_{n}$ values well within consistency threshold, suggesting a systematic effect probably due to a scaling factor in the calibration process. When data from Labs G and L are taken into consideration, an inconsistency in part of their acquired spectra was spotted in the $800-900 \mathrm{~nm}$ and $900-1100 \mathrm{~nm}$ band, respectively. This result is apparently in contradiction with the low W-by-W average differences shown in Figure 2(b) by Labs L and G, probably due to the wavy spectra so that the $\mathrm{W}$-by-W differences compensate over the entire wavelengths band. The same approach applied to Lab C data, confirmed highly divergent results due to instrument non-linearity or very poor calibration standards or procedures. Lab C recal data are relative to the recalibration exercise described in chapter 4. 


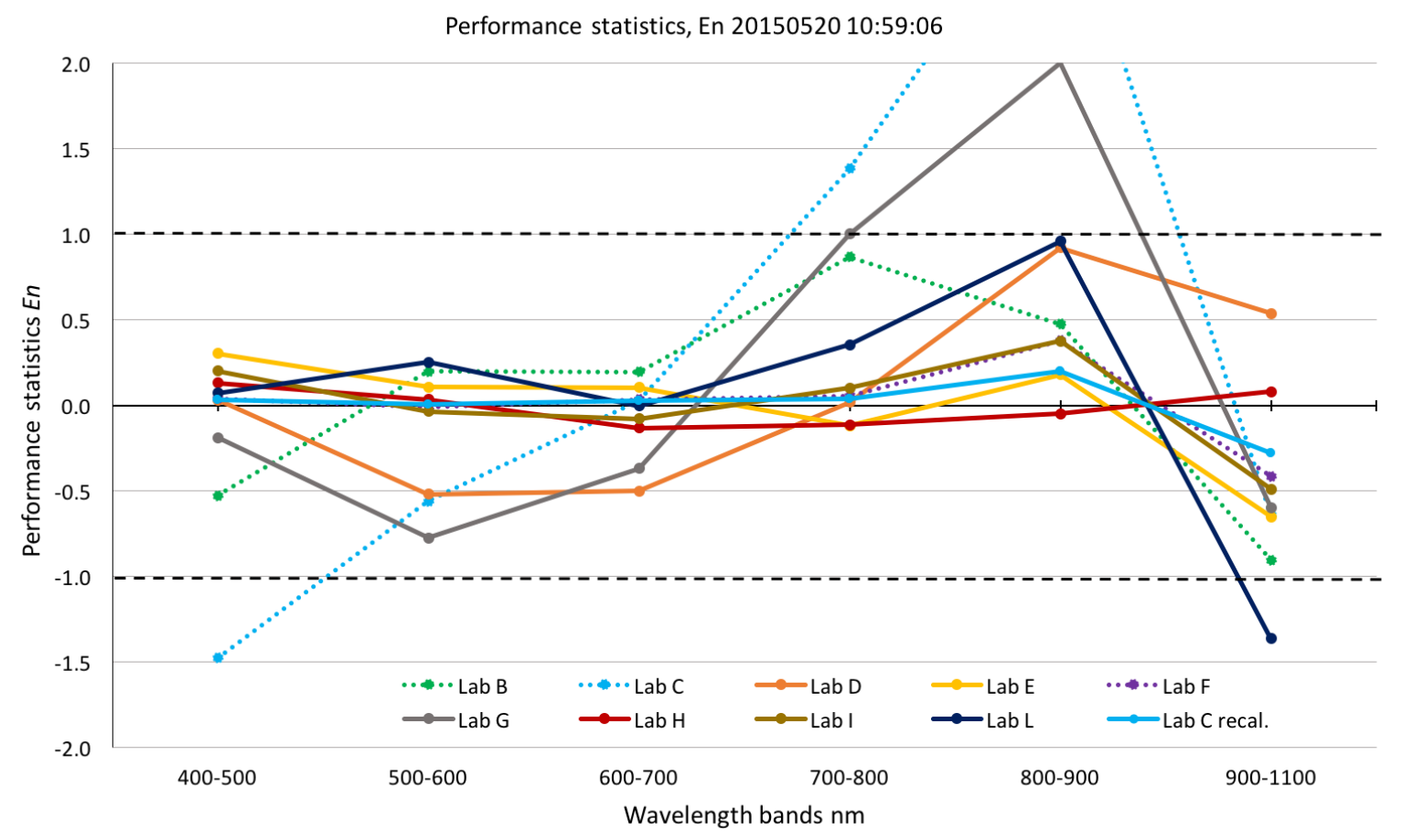

Figure 4: Performance statistics results using Equation (1). Percent spectral irradiance contents, as integrated in $100 \mathrm{~nm}$ bins from 400 to $900 \mathrm{~nm}$ plus a $200 \mathrm{~nm}$ bin from 900 to $1100 \mathrm{~nm}$, computed from spectra measured by various partner's instruments, are compared with those of the reference instrument and related to declared/assigned measurement uncertainty. Dashed lines at $\boldsymbol{E}_{\boldsymbol{n}} 1$, -1 represent acceptance/consistency limits. Results from the recalibration of the Lab C instruments are also reported (Cfr. Chapter 4).

Extending and applying the performance statistics $E_{n}$ number analysis to the twenty-eight stable spectra acquired on $19^{\text {th }}$ and $20^{\text {th }}$ of May 2015 gives us further information on instruments' time stability.

Figure 5 and Figure 6 summarize the daily average results; as in the analysis reported in Figure 4, the \pm 1 acceptance limits are set estimating $U L a b_{i}$ and Uref to be $5 \%$ and $3 \%$, respectively. Lab $\mathrm{C}$ recal data on both Figures are relative to the recalibration exercise described in the next chapter 4 . The $E_{n}$ number results do not change very much from one day to another; in both cases the same instrument systems (Labs B, D, E, F, H and I) with all deviation values within the acceptance limits (i.e. with deviation values coherent with the estimated uncertainty) reported in Figure 4 are also within \pm 1 limits in Figure 5 and Figure 6. This allows assessing the reproducibility of those systems over two days as they were dismounted overnight and remounted the day after.

The instrument system belonging to Lab L, which measured only on the $20^{\text {th }}$, confirmed to have one point (900-1100 nm band) out of the acceptance limits both in a specific measurement of the day and on the daily average, highlighting possible spectroradiometer`s misbehaviour in the near infrared wavelength region. Also Lab C data confirm severe deviations in three wavelength bands, probably due to inaccurate calibration reference and procedure. Lab G data show a different behaviour on the $19^{\text {th }}$, where its results showed in Figure 5 are in accordance with the acceptance levels, compared to the $20^{\text {th }}$, where the system showed one point out of limits, and needs further analysis. 
Performance statistics, En, 20150519 daily average

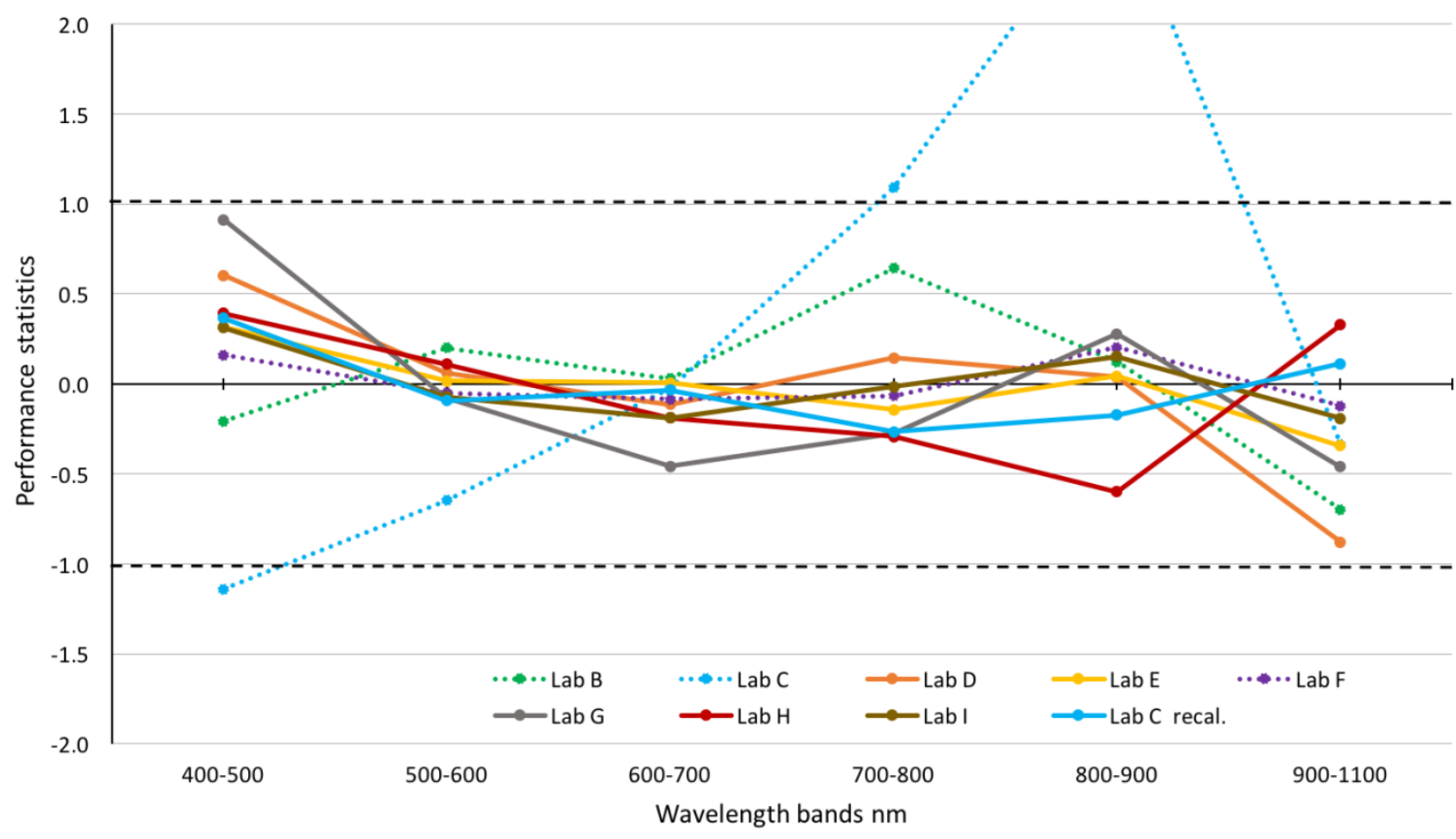

Figure 5: Average of the performance statistics results using Equation 1 for 1-day of stable measurements. Percent spectral irradiance contents, as integrated in $100 \mathrm{~nm}$ bins from 400 to $900 \mathrm{~nm}$ plus a $200 \mathrm{~nm}$ bin from 900 to $1100 \mathrm{~nm}$, computed from spectra measured by various partner's instruments, are compared with those of the reference instrument and related to declared/assigned measurement uncertainty. Dashed lines at $E_{n} 1,-1$ represent acceptance/consistency limits. Results from the recalibration of the Lab C instruments are also reported (Cfr. Chapter 4).

Performance statistics, En 20150520 daily average

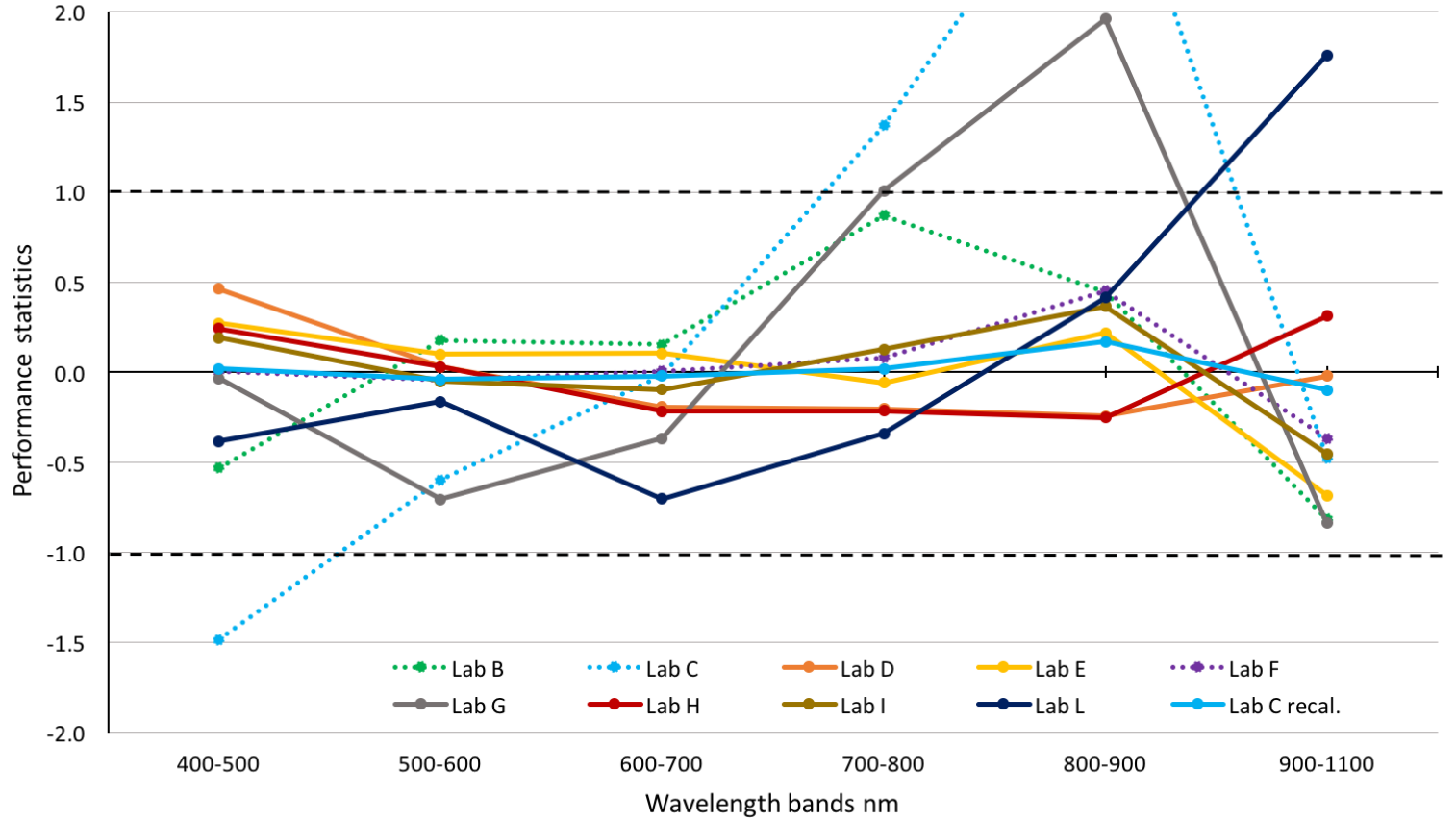

Figure 6: Average of the performance statistics results using Equation 1 for 1-day of stable measurements. Percent spectral irradiance contents, as integrated in $100 \mathrm{~nm}$ bins from 400 to $900 \mathrm{~nm}$ plus a $200 \mathrm{~nm}$ bin from 900 to $1100 \mathrm{~nm}$, computed from spectra measured by various partner's instruments, are compared with those of the reference instrument and related to declared/assigned measurement uncertainty. Dashed lines at $E_{n} 1,-1$ represent acceptance/consistency limits. Results from the recalibration of the Lab C instruments are also reported (Cfr. Chapter 4).

\subsection{Discussion}


The large amount of data resulting from the intercomparison must be dealt with diversified analysis methods to extract meaningful information about the characteristics and the behaviour of the spectroradiometers involved. The complexity of the instruments and measurements requires different analysis approaches trying to separate uncertainty and error components arising from systematic sources (e.g. calibration) from those arising from statistical sources, and those intrinsic to the instrument (e.g. temporal and thermal drift, internal stray light or grating second order effect).

In general, a careful scrutiny of the $\mathrm{W}$-by-W deviations calculated from the comparison with simultaneous acquired reference instruments spectra or from, in case of clear-sky conditions, a modelled SMARTS spectrum may give useful information on the instrument stability at short or long term; if more than one day data is available reproducibility information can also be inferred. However, more detailed analysis tools are necessary to better understand the behaviour of such complex systems.

In this work the use of performance statistics tools such as the $E_{n}$ number, allowed us to detect an instrument ( $\mathrm{Lab} \mathrm{H}$ ) with good intrinsic performance but with a systematic scaling factor, probably due to calibration (e.g.: wrong distance between standard lamp and target spectroradiometer, orientation or misalignment, drift of the reference lamp,...).

Moreover, the analysis on selected wavelength bands may give information about instrument behaviour on specific wavelength ranges. This is the case for Lab L, exhibiting $E_{n}$ values within the acceptance limits apart from the near infrared wavelength band (Figure 4 and Figure 6). The same approach applied over two days of measurements gave also a warning about the reproducibility of Lab $\mathrm{G}$ instrument that shows all data within the acceptance limits in Figure 5, but exhibits one point out of the acceptance limits the day after (see Figure 6).

\section{In-situ calibration of secondary instruments}

In addition to serve as a comparison of the performance of different instruments, the spectrometer intercomparison could be used to perform an in-situ calibration of secondary instruments based on the measurements made by primary instruments with traceability to a standard lamp (e.g. for laboratories not having access to traceable standards). This process is illustrated with the results of Lab C, which showed the poorest performance in terms of spectral distribution measurements (see section 3.2).

The spectra taken on the $20^{\text {th }}$ at 10:59 are used as the re-calibration point (Figure 2). A new calibration curve for the instrument of Lab $\mathrm{C}$ is calculated based on the spectra measured by Lab A and F as:

$$
R_{N e w}=R_{O l d} \frac{G_{L a b A}+G_{L a b F}}{2 G_{L a b C}}
$$

Where: $R_{N e w}$ and $R_{\text {Old }}$ are the new and old instrument calibration curves of Lab C, respectively; and $G_{L a b C}, G_{L a b A}$ and $G_{L a b F}$ the irradiances measured by Lab C, A and F, respectively. This equation assumes that the instrument properties - bandpass, slit function 
and straylight behaviour - are identical for the instruments involved. If that is not the case, the uncertainty of the new calibration curve will be higher.

Figure 7 shows the new calibration curve alongside the old one. As it can be seen, the original calibration was underestimating the solar irradiance in both the short and the long wavelength bands, while it was comparable in the intermediate band. This trend of having the largest error near the edges of the sensitivity range suggests that the reason for the poor performance of Lab C's instrument might be related with an incorrect account of the background signal of the spectrometer, either during the calibration or during the intercomparison. The ripples in the short and long wavelength ranges are an artefact of the recalibration process. They can be related with differences in the instrument bandpass and the convolution and interpolation process described in Section 2, which have a large impact in the spectral regions with narrow atmospheric absorption bands. As mentioned, these differences will result in a higher uncertainty of the new calibration curve in these spectral regions.

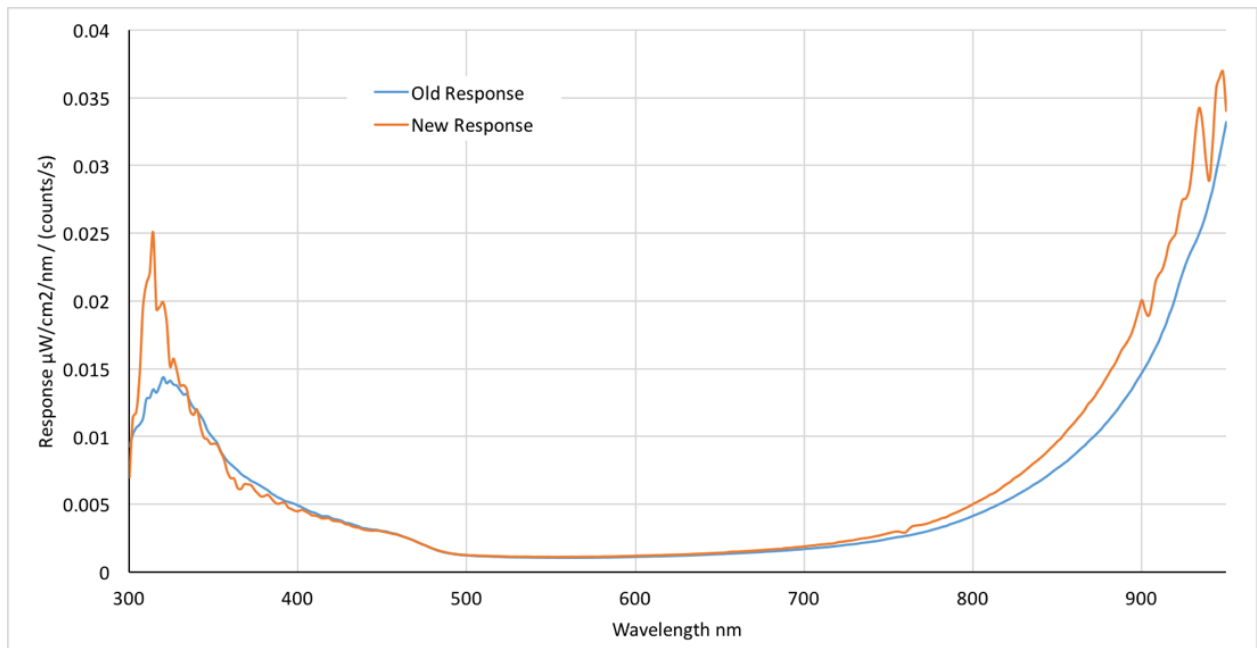

Figure 7: Old and New calibration curve of the instrument of Lab C after in-situ recalibration based on the measurements of Lab A and Lab F on the 20th, 10:59.

This new calibration curve is then used to re-calculate all spectra measured by Lab C during the intercomparison: the new spectra, duly convoluted and interpolated, have been referred to as "Lab C recal" in the previous sections.

The 1-Day average W-by-W differences and standard deviations values calculated with respect to Lab A spectra for the measurements of the $19^{\text {th }}$ and $20^{\text {th }}$ are show in Table 2 . As it can be seen, the re-calibration process results in an overall improvement of all the re-processed measurements compared to Lab A, not only at the calibration point. In particular, the strong reduction of the standard deviation indicates a good stability of the instrument over the time of the intercomparison. The relative spectral distribution analysed in Section 3.2 has also improved with the new calibration. In all situations (Figures 4, 5 and 6 ), Lab C performance statistics have changed from being well outside the \pm 1 acceptance limits to less than \pm 0.5 in all spectral bands. However, similar performance might not be obtained when measuring light sources with strongly different spectral irradiance 
distribution due to limitations deriving from different bandpass, slit function and straylight behaviour among involved instruments. This procedure represents, however, an acceptable trade-off between easy implementation and a higher calibration uncertainty.

This in-situ recalibration of secondary instruments not only provides a means of having an instrument calibration curve traceable to a primary instrument: the information can be very valuable for Lab $\mathrm{C}$ to identify the critical aspects of their calibration process that need to be revised and improved in order to reach acceptable standards for PV applications.

\section{Results impact on a PV cell calibration exercise}

One of the aims of the intercomparison is to establish equivalence confidence limits about the measurements of solar spectrum useful for the PV community. An easy to understand way to establish such an equivalence confidence limit, is to determine how much the use of solar spectra measured simultaneously by various partners may influence the output of the short circuit current (Isc) calibration of a reference solar cell.

Several primary calibration methods are described and reported in the annex A of the IEC standard referred in [10]. Specifically, a slightly modified version of the global sunlight method will be used in the following. The calibration of a PV device at standard test conditions (STC) entails, among others, the spectral mismatch correction [6] to the standard spectrum, which is mainly AM1.5G [7] for terrestrial applications. This correction is performed by applying a mismatch correction factor (MM) accounting for the difference in spectral responsivities between the reference device and the testing one, and for the relative spectral difference between testing light source and standard spectrum.

The knowledge of the actual spectrum of the solar radiation impinging on the surface of a PV reference cell is one of the necessary parameters for its calibration, the others being the spectral responsivities of the solar cell under calibration and of the reference device used to measure the solar radiation intensity, and the AM1.5G reference spectrum. All these input parameters allow computing, the following spectral mismatch correction factor.

$$
M M=\frac{\int S R_{r e f}(\lambda) G_{r e f}(\lambda) d \lambda}{\int S R_{r e f}(\lambda) G_{\text {meas }}(\lambda) d \lambda} \frac{\int S R_{d u t}(\lambda) G_{\text {meas }}(\lambda) d \lambda}{\int S R_{d u t}(\lambda) G_{\text {ref }}(\lambda) d \lambda}
$$

In eq. $3 S R_{\text {ref }}(\lambda)$ represents the spectral responsivity of the reference device (which is assumed to be constantly 1 for broadband radiometers), $G_{r e f}(\lambda)$ is the spectral irradiance of the AM1.5G reference spectrum, $S R_{d u t}(\lambda)$ represents the spectral responsivity of the reference solar cell to be calibrated, and $G_{\text {meas }}(\lambda)$ is the actual solar spectrum as measured at the time of calibration.

It is worth noticing that $\mathrm{MM}=1$ when $S R_{\text {ref }}(\lambda)=S R_{\text {dut }}(\lambda)$ or $G_{\text {ref }}(\lambda)=G_{\text {meas }}(\lambda)$. Therefore, using a broadband radiometer as reference device the deviations between the measured spectrum and the standard AM1.5G are highlighted, due to the large spectral responsivity differences between reference device and device under test.

In this exercise we calculated MMs values for different simultaneously measured spectra by four (Labs A, E, F, H) of the 'best performing` spectroradiometers at different times during the first measurement day (19/05/2015). According to [10], the integrals of eq. 3 must be computed in the working spectral range of the broadband radiometer used as reference 
device (e.g. 250 to $4000 \mathrm{~nm}$ ). However, for this exercise we limited the integrals range from 300 to $1100 \mathrm{~nm}$ in order to accommodate the measuring bandwidth of all the involved instruments. Moreover, here we are interested in highlighting the difference in the relative spectral shape among the acquired spectra by different partners; hence, the bandwidth reduction will not affect, in the considered wavelength range, the comparison results.

Figure 8 shows the data used to calculate the MM factors at four different times during a measurement day. It includes the spectral responsivity of the reference cell under calibration, the solar spectra acquired by four spectroradiometers at different times of the day and the AM1.5G reference spectrum. For display purposes Y-axes arbitrary unit has been chosen in order to shift plots.

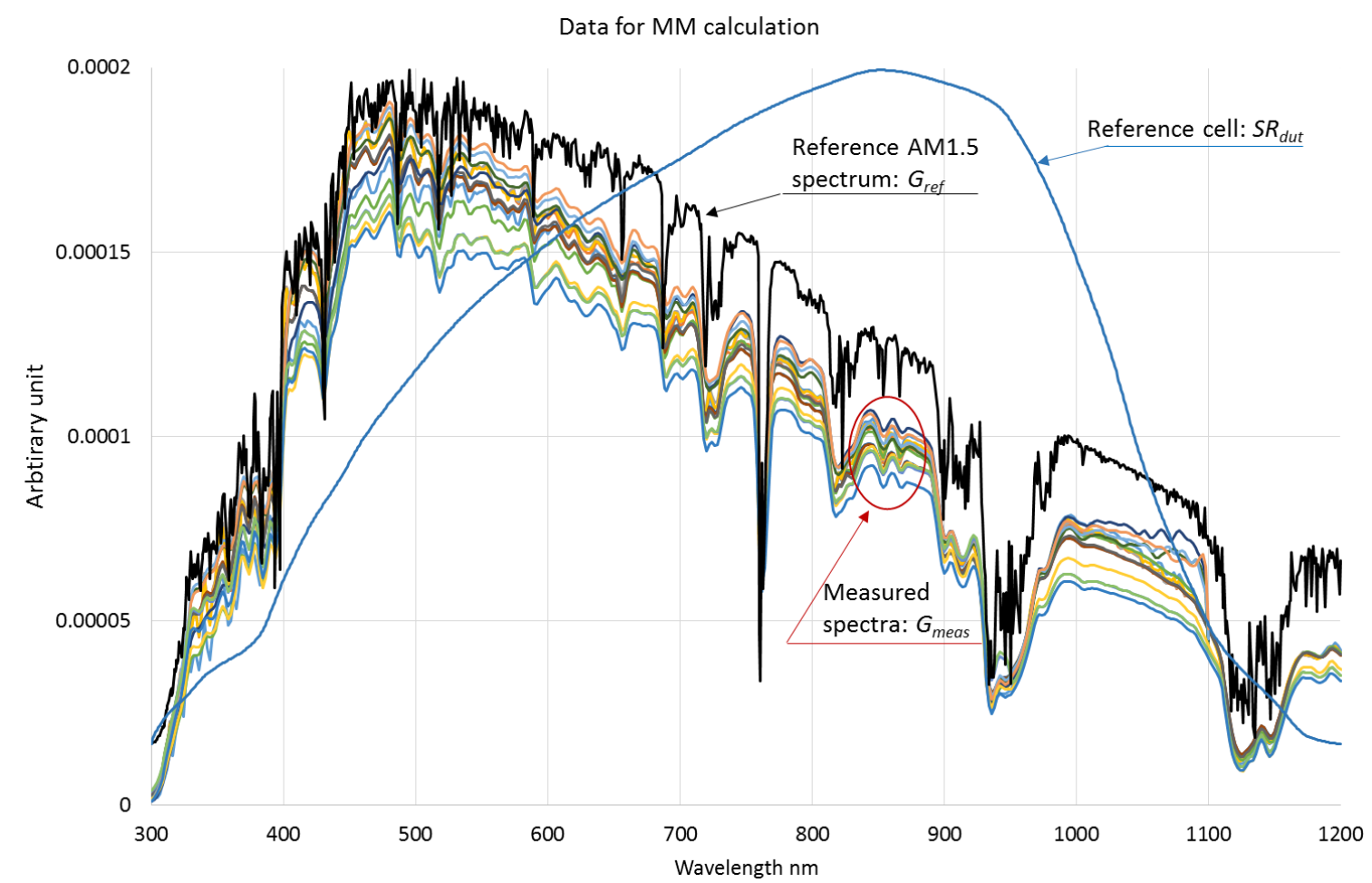

Figure 8: Reference AM1.5G spectrum, $G_{r e f}(\lambda)$, measured spectra at different time of the day by four spectroradiometer systems, $G_{\text {meas }}(\lambda)$, and spectral responsivity of calibrating solar cell, $S R_{d u t}(\lambda)$, data used in Equation 3 to compute MM values reported in table 3 .

MM computation results are reported in Table 3. The peak to peak (pk-pk) differences of the computed MM ranges from $0.4 \%$ for a set of stable simultaneously acquired spectra at the beginning of the measurement day and increases to max $1 \%$ later during the day, probably due to a slight drift of Lab $\mathrm{H}$ instrument.

\begin{tabular}{lcccc} 
& \multicolumn{4}{c}{ Spectra acquisition time on May the 19th } \\
& $10: 27$ & $12: 08$ & $12: 18$ & $14: 47$ \\
\hline MM Lab A & 1.003 & 1.019 & 1.018 & 1.018 \\
MM Lab E & 1.007 & 1.021 & 1.022 & 1.019 \\
MM Lab F & 1.003 & 1.020 & 1.020 & 1.019 \\
MM Lab H & 1.004 & 1.012 & 1.012 & 1.011 \\
\hline MM pk-pk \% difference & 0.4 & 0.9 & 1.0 & 0.8
\end{tabular}


Table 3: Spectral mismatch correction factors as computed using equation 3 with a set of simultaneously acquired solar spectra at different time of the day by four of the spectroradiometer systems participating to the intercomparison.

The MM values for the two reference labs present at the intercomparison (Lab A and Lab F) are in good agreement to within $0.2 \%$ pk-pk for the whole measurement day. These results confirm the importance of a correct, reliable and traceable solar spectrum measurement for high precision PV devices calibration as a $1 \%$ difference in MM factor turns to a $1 \%$ difference in the final $\mathrm{I}_{\mathrm{sc}}$ calibration value.

Although it is possible to reduce the influence of MM correction factor, and hence of its uncertainty [11], (e.g. choosing the calibration time and location in order to be as close as possible to AM1.5G condition), it is likely that a significant part of the $1 \%$ to $3 \%$ spread reported in the Isc values for PV modules $[12,13]$ and cells $[14,15]$ calibration round robin is due to the uncertainty in measuring the spectrum of the sun or of the solar simulators.

\section{Conclusions}

A spectroradiometers intercomparison was performed among ten European scientific and industrial partners. The intercomparison aimed at exchanging and comparing instrument calibration procedures and measurement capabilities, at establishing equivalence figures for solar spectra measurement, and at putting in practice lessons learnt from previous editions. The large variety of manufacturers and principles of operation of the instruments involved in the intercomparison are a good cross section of today's most used spectroradiometers in the PV community. Different and complementary data analyses were applied to the measured spectra so that partners can have comprehensive knowledge about their instrument's behaviour, calibration, and measurement procedures. The analyses of the results showed W-by-W average differences lying within an interval of $\pm 3.2 \%$ for two whole days of measurement, for a total of twenty-eight stable spectra. Performance statistics $E_{n}$ number analysis applied to the same acquisition days showed that in all the considered wavelength bands, six spectroradiometers have their output consistent with declared measurement uncertainty on both days. One had $E_{n}$ results consistent with the declared uncertainty on one day and showed partial inconsistency on the other day. One other system showed larger deviation from \pm 1 acceptance limits on both days.

Absolute and relative spectral irradiance comparison methodologies proved to be a good approach to separate instrument non-linearity and distortion effects, from systematic effects due to calibration or due to drift with time and/or temperature. In addition to serve as a comparison of the performance of different instruments, the spectrometer intercomparison can be used to perform an in-situ calibration of secondary instruments for measuring natural sunlight based on the measurements made by primary instruments with traceability to a standard lamp. Such an exercise proved that correcting acquired spectra according to recalibration significantly improved the agreement with reference instruments and can be valuable for identifying critical aspect of calibration.

When quantifying the impact of using different simultaneously acquired spectra on the Isc calibration of a PV reference cell, we found a maximum $1 \%$ pk-pk figure for spectral mismatch calculation using data from four different laboratories, reducing to $0.2 \%$ pk-pk 
when data from two reference laboratories were considered.

\section{ACKNOWLEDGMENT}

The intercomparison has been partially funded from the SolCell project through the EMRP contract. ENG51- REG3, and from the project PhotoClass through the contract EMRP ENG55. The EMRP is jointly funded by the European Union and the EMRP participating countries within EURAMET.

Appendix A:

SMARTS (Simple Model of the Atmospheric Radiative Transfer of Sunshine) is used to create synthetic spectra for all the days and times of the intercomparison [16]. Real time atmospheric parameters (ambient temperature, surface pressure, precipitable water and humidity) were measured on site or retrieved from public repositories of atmospheric data measured at a weather station in the nearby Madrid-Barajas airport [17-19]. Other parameters needed for the simulation (such as albedo or aerosol model) were fixed throughout all simulations. In Appendix B there is an example of a SMARTS input file for the 9:17 measurement of the $20^{\text {th }}$ of May 2015 .

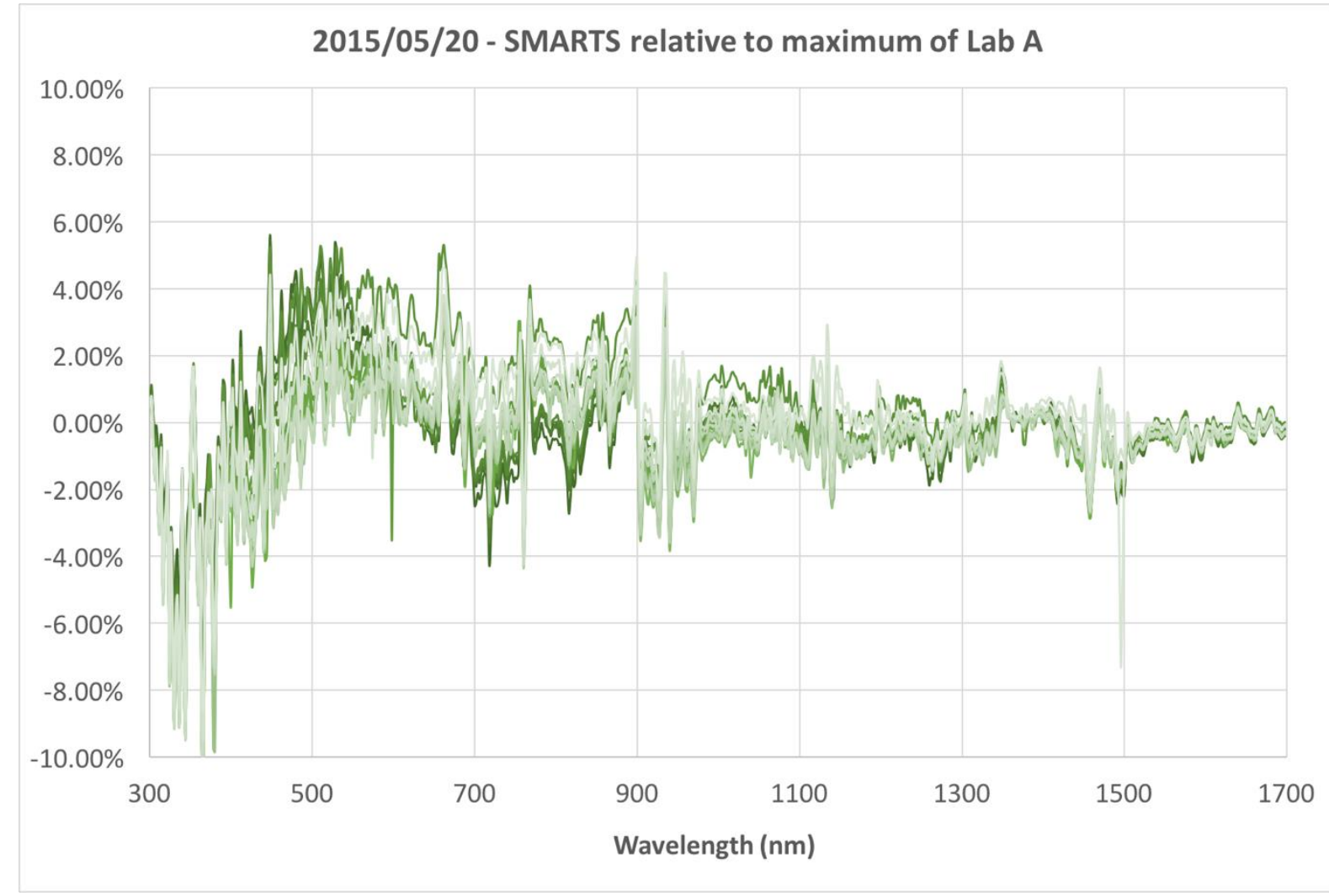

Figure 9: Wavelength-by-wavelength difference of SMARTS synthetic spectra with respect Lab A for all spectra measured on the $20^{\text {th }}$ of May (21 in total).

Figure 9 shows the wavelength-by-wavelength difference of SMARTS synthetic spectra 
with respect to Lab A for all spectra measured on the $20^{\text {th }}$ of May (21 in total). A consistent difference less than $5 \%$ at all wavelengths and less than $2 \%$ for the near infrared region $(>900 \mathrm{~nm})$ can be observed independently of the spectrum. These values are on the same order, or better, than those obtained experimentally by some of the labs shown in Figure 1.

Appendix B:

Example of input file for SMARTS. See SMARTS documentation for a full explanation of the meaning of each "Card" entry.

'2015-05-20_09-17_SMARTS' !Card 1

1 ! Card 2

952.60 .6250 .0 !Card 2a

1 ! Card 3

'USSA' !Card 3a

0 !Card 4

0.6984846907675195 !Card 4a

1 !Card 5

0 !Card 6

2 !Card 6a

370 !Card 7

0 !Card 7a

'S\&F_RURAL' !Card 8

0 !Card 9

0.085 !Card 9a

9 !Card 10

1 !Card 10b

9 -999 -999 !Card 10c

280400411367 !Card 11

2 !Card 12

28040040.5 !Card 12a

4 !Card $12 \mathrm{~b}$

$6,7,8,9$ !Card 12c

1 !Card 13

12.54 !Card 13a

1 !Card 14

11700170024 !Card 14a

0 !Card 15

0 !Card 16

3 !Card 17

20155208.22540 .4966 -3.462 0 !Card 17a 


\section{References:}

1. ISO/IEC 17025:2005, General requirements for the competence of testing and calibration laboratories, International Organization for Standardization, ISO Central Secretariat, Chemin de Blandonnet 8, CP 4011214 Vernier, Geneva, Switzerland

2. Guide to meteorological instruments and observations, WMO-No-8 Secretariat of the World Meteorological Organisation, 2006, Geneva, Switzerland

3. Galleano R., Zaaiman W., Virtuani A., Pavanello D., Morabito P., Minuto A., Spena A., Bartocci S., Fucci R., Leanza G., Fasanaro D., and Catena M. (2014), Intercomparison campaign of spectroradiometers for a correct estimation of solar spectral irradiance: results and potential impact on photovoltaic devices calibration, Prog. Photovolt: Res. Appl., 22, pages 1128-1137, doi: 10.1002/pip.2361

4. Galleano, R, Zaaiman, W, Strati, C, Bartocci, S, Pravettoni, M, Marzoli, M, Fucci, R, Leanza, G, Timò, G, Minuto, A, Catena, M, Aleo, F, Takagi, S, Akiyama, A, Nuñez, R, and Belluardo, G (2015), Second international spectroradiometer intercomparison: results and impact on PV device calibration. Prog. Photovolt: Res. Appl., 23, 929-938. doi: 10.1002/pip.2511

5. Finsterle, W., (2011), WMO International Pyrheliometer Comparison IPC-XI Final Report,WMO IOM Report No., 108

6. ISO/IEC 60904-7 Photovoltaic devices - Part 7: Computation of spectral mismatch error introduced in the testing of a photovoltaic device, International Organization for Standardization, ISO Central Secretariat, Chemin de Blandonnet 8, CP 4011214 Vernier, Geneva, Switzerland

7. ISO/IEC 60904-3 Photovoltaic devices - Part 3 Measurement principles for terrestrial photovoltaic (PV) solar devices with reference spectral irradiance data, International Organization for Standardization, ISO Central Secretariat, Chemin de Blandonnet 8, CP 4011214 Vernier, Geneva, Switzerland

8. ISO/IEC 60904-9:2007 Photovoltaic devices - Part 9: Solar simulator performance requirements, International Organization for Standardization, ISO Central Secretariat, Chemin de Blandonnet 8, CP 4011214 Vernier, Geneva, Switzerland

9. ISO/IEC 17043:2010 Conformity assessment-General requirements for proficiency testing, ISO Central Secretariat, Chemin de Blandonnet 8, CP 4011214 Vernier, Geneva, Switzerland

10. ISO/IEC 60904-4 Photovoltaic devices - Part 4: Reference solar devices Procedures for establishing calibration traceability, International Organization for Standardization, ISO Central Secretariat, Chemin de Blandonnet 8, CP 4011214 Vernier, Geneva, Switzerland

11. H. Field and K. A. Emery, "An Uncertainty Analysis of the Spectral Correction Factor", Proc. of 23rd IEEE Photovohaic Specialists Conference. 1993, pp. 1 180-1187

12. Hishikawa, Y., Liu, H., Hsieh, H.-H., Inoue, T., Kim, K.-S., Limsakul, C., Kim, S.W., Ninae, R. and Morita, K. (2013), Round-robin measurement intercomparison of c-Si PV modules among Asian testing laboratories. Prog. Photovolt: Res. Appl., 21: 1181-1188. doi:10.1002/pip.2255

13. D Dirnberger et al 2014 Meas. Sci. Technol. 25 105005, doi: 10.1088/0957- 
$\underline{0233 / 25 / 10 / 105005}$

14. P. Manshanden et aliis, Round Robins of Solar Cells to Evaluate Measurement Systems of Different European Research Institutes, Proceedings 28th European Photovoltaic Solar Energy Conference and Exhibition, 30 September - 4 October 2013, Paris, France, pg. 861

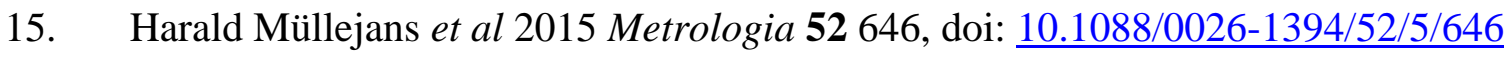

16. SMARTS webpage. Software and user manual. http://www.nrel.gov/rredc/smarts/ . Last visit: January 2016.

17 G. Martinez Fuente (INTA, Spain), Ambient temperature and humidity measured in-situ at INTA, Private communication.

18 Atmospheric pressure measured at Madrid-Barajas airport. Data retrieved from www.ogimet.com.

19 Precipitable water obtained from the Madrid site of AERONET, http://aeronet.gsfc.nasa.gov/ 